\title{
Alter
}

Revue de phénoménologie

$20 \mid 2012$

Eros

\section{Jonna Bornemark \& Marica Sà Cavalcante Schuback (eds.), Phenomenology of Eros}

avec la collaboration de Peter Trawny, Elisabeth B. Sikes, Anna-Lena Renqvist, Agnès Pigler, Jason Wirth, Françoise Dastur, Nicholas Smith, Helena Dahlberg, Carl Cederberg, Lisa Folkmarson Käll, Södertörn Philosophical Studies 10, 288 pages.

\section{Philippe Cabestan}

\section{OpenEdition}

\section{Journals}

Édition électronique

URL : http://journals.openedition.org/alter/1136

DOI : $10.4000 /$ alter.1136

ISSN : 2558-7927

\section{Éditeur :}

Association ALTER, Archives Husserl (CNRS-UMR 8547)

\section{Édition imprimée}

Date de publication : 1 décembre 2012

Pagination : 223-227

ISBN : 978-2-95-223748-2

ISSN : 1249-8947

\section{Référence électronique}

Philippe Cabestan, « Jonna Bornemark \& Marica Sà Cavalcante Schuback (eds.), Phenomenology of Eros », Alter [En ligne], 20 | 2012, mis en ligne le 01 juillet 2019, consulté le 24 septembre 2020. URL http://journals.openedition.org/alter/1136 ; DOI : https://doi.org/10.4000/alter.1136 
PHENOMENOLOGY OF EROS, JONNA BORNEMARK \& MARICA SA CAVALCANTE SCHUBACK (EDS.), AVEC LA COLLABORATION DE PETER TRAWNY, ELISABETH B. SIKES, ANNA-LENA RENQVIST, AGNES PIGLER, JASON WIRTH, FRANÇOISE DASTUR, NICHOLAS SMITH, HELENA DAHLBERG, CARL CEDERBERG, LISA FOLKMARSON KÄLL, SÖDERTÖRN PHILOSOPHICAL STUDIES 10, 288 PAGES.

Philippe Cabestan

Ce volume rassemble pour l'essentiel les contributions d'un colloque organisé à l'université de Södertörn (Suède) en 2006. Dans leur introduction, les éditrices, J. Bornemark et M. Sà Cavalcante Schuback, rappellent l'importance et la diversité des travaux que des phénoménologues comme Max Scheler, Ortega y Gasset, Eugen Fink, Simone de Beauvoir, Ludwig Binswanger ainsi qu'Emmanuel Levinas et Jean-Luc Marion ont implicitement ou explicitement consacré au phénomène érotique. Comment la phénoménologie aurait-elle pu laisser de côté cette dimension fondamentale de l'intersubjectivité ou plutôt de l'intercorporéité ! Reste à savoir si, en se risquant sur un terrain tout à la fois si bien et si mal fréquenté, elle est demeurée fidèle à elle-même et à l'ambition qui l'anime sans céder aux sirènes de la spéculation.

L'un des mérites de cette publication tient tout d'abord à son souci d'ouverture historique et à sa volonté d'accorder une large place à la manière dont l'antiquité a abordé et peut-être façonné le phénomène érotique. De ce point de vue, comme le montre P. Trawny, il convient de situer l'amour platonicien en rupture avec l'Erôs que mettent en scène les poètes tragiques comme Sophocle dans Antigone et Euripide dans Hippolyte. Alors que la tragédie tient Erôs pour un tyran qui n'a jamais connu de loi, et auquel nul n'échappe, y compris les dieux, 
Platon pour sa part lui reconnaît une tout autre nature tout en accordant que Erôs conduit aux pires excès lorsqu'il règne dans une âme que domine l'injustice. Réinterpréter psychologiquement, au sens étymologique du terme, Erôs se tient désormais au cœur de l'aspiration philosophique à la contemplation des réalités intelligibles. Ajoutons toutefois, avec A.-L. Renqvist, que sa nature n'est pas purement théorique puisqu'il est essentiellement fécond et qu'il a pour objet non seulement la beauté mais aussi la naissance et la procréation. Ces premiers éléments permettent alors de saisir ce qui, sur ce chapitre, sépare Platon de son disciple Plotin au point que, selon A. Pigler, surgisse sous la plume du philosophe néo-platonicien une «nouvelle métaphysique »: si, comme nous venons de le voir, le siège de l'amour est pour Platon dans l'âme et nulle part ailleurs, pour Plotin, en revanche, Erôs demeure originellement dans l'Un. Erôs est alors la condition de la procession comme de la conversion qui permettent la constitution de l'Intelligible et du Sensible. En raison de sa nature comme de son origine, l'amour n'est plus cette semi-divinité platonicienne mais il est de part en part divin.

La seconde partie de l'ouvrage rassemble des travaux qui $s$ 'inscrivent explicitement dans une perspective phénoménologique. Il faut rappeler à ce propos que Husserl lui-même, et de manière quelque peu inattendue, initie dans ses manuscrits sur l'intersubjectivité une description prometteuse de la jouissance sexuelle, dont il souligne notamment la dimension fondamentalement intersubjective. De ce point de vue, écrit-il, " elle ne peut avoir lieu seule, et les deux partenaires ne peuvent pas non plus, en jouissant, prendre conscience chacun pour soi de la jouissance ; ils peuvent bien plutôt être là l'un pour l'autre, jouissant l'un avec l'autre et l'un par l'autre, et aspirant à la jouissance l'un de l'autre ; aspirant tout uniment, dans cet état de quasi-fusion, à la jouissance, ils peuvent former l'unité d'une communauté de jouissance $»^{1}$. Il n'est pas difficile de saisir la parenté d'une telle description - même si J.-P. Sartre ignorait ces textes du Nachlass - avec celle de la chair et du désir charnel dans L'Être et le néant, qu'étudie H. Dahlberg. Car, nonobstant son irrémédiable échec, le désir sartrien est fondamentalement intersubjectif et vise à travers la caresse l'empâtement réciproque des consciences.

L. Folkmarson Käll retrouve à son tour cette dimension intersubjective de la sexualité dans La Mort à Venise et la contemplation

\footnotetext{
${ }^{1}$ E. Husserl, Sur l'intersubjectivité, trad. fr. N. Depraz, Paris, PUF, 2001, T.II, texte No 9, §8, p. 277. Cette description de la jouissance est implicitement reprise par J.-L. Marion dans Le phénomène érotique, Paris, Grasset, 2003, p. 201.
} 
érotique du jeune Tadzio par le vieux von Aschenbach. Soulignant le caractère paradigmatique de la perception érotique selon MerleauPonty, l'auteur rappelle, d'une part, que celle-ci repose sur une intentionnalité corporelle non représentative ou intentionnalité opérante qui donne accès au monde ; et, d'autre part, que le désir qui anime en l'occurrence le regard caressant du vieil homme n'est pas totalement désintéressé, s'il est vrai qu'il convoite en retour le regard caressant de l'autre. Ainsi le désir de voir (de toucher) est-il désir d'être vu (touché), bref d'être voyant-visible (touchant-touché). De même, loin d'être " un plaisir solitaire comme le manger et le boire $»^{2}$, la volupté levinassienne présuppose une communauté au sein de laquelle le désir est désir de l'autre. Parce que le reproche fut adressé à Sartre comme à Levinas, on peut évidemment se demander avec J. Bornemark si ces différentes descriptions, ainsi que celles de M. Henry ou J.-L. Marion, ne sont pas les feuilles de vigne d'une conception à vrai dire phallocentrique de la sexualité ("a male-centric, heterosexist, partriarchal variant of sexuality") ${ }^{3}$. Dans son article, "Accusing the erotic Subject in Levinas", C. Cederberg admet pour sa part que les critiques d'une Luce Irigaray à l'encontre de Levinas ne sont pas sans fondement même si elles reposent sur une relative incompréhension de la pensée profonde du philosophe qui n'a jamais prétendu en ce domaine à une quelconque forme de neutralité sexuelle. Tandis que J. Borneman, de manière plus violente et sans doute plus contestable, reproche à J.-L. Marion d'associer la femme à l'inactivité et à la passivité au point d'en faire, au sein la relation sexuelle, la victime d'un viol (a rape-victim) ${ }^{4}$. Plus fondamentalement, en opposition à une séparation trop radicale de la chair et du corps par J.-L. Marion, J. Borneman se propose d'envisager la relation érotique et notamment l'orgasme comme une expérience-limite de destruction des frontières entre l'amant et l'aimé, la chair et le corps, l'immanence et la transcendance.

Nous ne voudrions pas achever cette recension sans évoquer deux contributions inspirées par l'œuvre de Heidegger. Mais, à vrai dire, peut-on parler d'une conception heideggérienne de l'amour ou encore d'un heideggerian love? Telle est du moins la conviction de M. Sà

\footnotetext{
2 E. Levinas, Le temps et l'autre, Paris, PUF, 1983, p. 82.

${ }^{3}$ Phenomenology of Eros, p. 247.

${ }^{4}$ Phenomenology of Eros, p. 258. Il nous semble au contraire que J.-L. Marion ne cesse d'affirmer la réciprocité du processus érotique et prend soin de ne pas subordonner sa description de la jouissance au stéréotype de la double opposition du masculin-actif et du féminin-passif. Il écrit par exemple : "L'avancée et le retrait d'une chair attend l'avancée et le retrait de l'autre aussi longtemps qu'elles peuvent (se) ressentir », J.-L. Marion, Le phénomène érotique, op. cit., p. 206.
} 
Cavalcante Schuback qui trouve dans les rares paroles de Heidegger à ce sujet la possibilité d'approfondir le lien qui unit l'amour et la philosophie. En outre, l'auteur précise en quel sens Heidegger fait sienne la conception augustinienne de l'amour - que l'on retrouve dans sa correspondance avec $\mathrm{H}$. Arendt - que résume la sentence : volo ut $\mathrm{sis}^{5}$; ainsi que la nature du fossé qui le sépare du psychiatre L. Binswanger dont les Grundformen (1942) entendent compléter l'analytique existentiale en y ajoutant l'amour au côté du souci. C'est oublier, comme le souligne également $\mathrm{F}$. Dastur, que l'amour a déjà toute sa place au sein de l'analytique existentiale et qu'il trouve son fondement ontologique dans la structure du souci. De ce point de vue, la séduction n'est pas à l'amour ce que l'apparence est à la réalité, mais elle en est une modalité dégradée qu'il convient de comprendre également à partir du souci et de la déchéance du Dasein dans l'inauthenticité. En d'autres termes, la séduction correspond à la manière dont nous nous comportons tout d'abord et le plus souvent de sorte qu'au lieu d'aimer nous voulons être aimé, c'est-à-dire attirer, plaire et dominer l'autre.

Sans doute ces quelques lignes témoignent-elles trop rapidement de la qualité et de la richesse des analyses proposées dans cet ouvrage dont la lecture offre une bonne idée des recherches phénoménologiques en ce domaine. Permettons-nous en conclusion de formuler cependant deux regrets qui touchent l'un comme l'autre à la nature de ce qui est en question. En effet, force est de constater que le phénomène érotique demeure dans cet ouvrage relativement indéterminé. C'est bien pourquoi, comme l'écrivent J. Bornemark et M. Sà Cavalcante Schuback dans leur introduction, "des phénoménologies de l'amour, du désir, de la séduction, de la passion, de la sexualité et de la différence sexuelle sont des phénoménologies de l'erôs et vice-versa ». Or, comme nous l'avons vu, à propos de la séduction et de l'amour chez Heidegger, n'y a-t-il pas également une différence fondamentale, jusque dans les gestes qu'ils commandent, notamment entre l'amour et le désir charnel ? En outre, on peut s'étonner de la "sagesse" des descriptions du phénomène érotique, qui semblent ignorer non seulement son socle biologique mais aussi l'extraordinaire diversité des pratiques - parfois criminelles - que suscite "tout d'abord et le plus souvent" Erôs chez le commun des mortels. Non sans humour et avec un esprit polémique que peu de

\footnotetext{
5 « être en proie à l'amour = être rabroué à son existence la plus propre. Amo, à savoir volo ut sis, a pu dire saint Augustin : je t'aime - je veux que tu sois ce que tu es ", M. Heidegger, Lettres et autres documents, 1925-1975, trad. fr. P. David, Paris, Gallimard, 2001, p. 36.
} 
ses contemporains lui connaissaient, Dominique Janicaud dénonçait dans la caresse levinassienne un "érotisme de vitrail $»^{6}$. Reconnaissons que, vingt et un ans plus tard, le phénomène érotique demeure relativement désincarnée.

${ }^{6}$ D. Janicaud, Le tournant théologique de la phénoménologie française, Paris, éditions de l'Eclat, 1991, p. 30 . 